Histoire de l'éducation

DEDUCATION

$122 \mid 2009$

L'enseignement supérieur

\title{
Avant-propos
}

\section{Emmanuelle Picard}

\section{OpenEdition}

Journals

Édition électronique

URL : https://journals.openedition.org/histoire-education/1937

DOI : 10.4000/histoire-education.1937

ISSN : 2102-5452

\section{Éditeur}

ENS Éditions

\section{Édition imprimée}

Date de publication : 1 avril 2009

Pagination : 5-10

ISBN : 978-2-7342-1151-8

ISSN : 0221-6280

Référence électronique

Emmanuelle Picard, « Avant-propos », Histoire de l'éducation [En ligne], 122 | 2009, mis en ligne le 27 octobre 2009, consulté le 20 mai 2021. URL : http://journals.openedition.org/histoire-education/1937 ; DOI : https://doi.org/10.4000/histoire-education.1937

(C) Tous droits réservés 


\title{
Avant-propos
}

\author{
Emmanuelle PICARD
}

Comparée à l'importante bibliographie dont bénéficient les autres niveaux d'enseignement, l'histoire de l'enseignement supérieur apparaît comme le parent pauvre de l'histoire de l'éducation en France. Si certains domaines ont été explorés en détail, comme la sociologie du corps enseignant ou l'histoire des disciplines universitaires, d'autres sont encore mal connus aujourd'hui. La célébration du centenaire, ou du bicentenaire, de certaines institutions a suscité des publications dont le volume et la richesse sont en rapport avec le prestige de ces institutions et les travaux antérieurs dont elles avaient fait l'objet ${ }^{1}$. Ainsi, l'École normale supérieure (rue d'Ulm) et l'École polytechnique ont toutes deux bénéficié en 1994 d'importantes opérations commémoratives, qui ont été l'occasion de multiples colloques, ouvrages collectifs et répertoires de sources ${ }^{2}$. C'est une exception dans la pauvreté historiographique générale.

Or, cette pauvreté est paradoxale si on la rapporte à l'influence du modèle universitaire napoléonien dans toute une partie des pays occidentaux

1 Pour ne pas alourdir le propos, nous renvoyons à la bibliographie des établissements d'enseignement supérieur mise en ligne sur le site du Service d'histoire de l'éducation à l'adresse : <http://www.inrp.fr/she/picard_biblio_etablissements_enseignement_superieur.htm>. La situation historiographique des différentes institutions est très hétérogène, les universités étant incontestablement les parents pauvres de la recherche.

2 Pascale Hummel, Anne Lejeune, David Peyceré, Pour une histoire de l'École normale supérieure. Sources d'archives (1794-1993), Paris, Archives nationales/Presses de l'ENS, 1995 ; Jean-François Sirinelli (dir.), École Normale Supérieure. Le Livre du Bicentenaire, Paris, Presses de l'ENS, 1994 ; Bruno Belhoste, Amy Dahan-Dalmédico, Dominique Pestre, Antoine Picon (dir.), La France des X. Deux siècles d'histoire, Paris, Économica, 1995. 
aux $\mathrm{XIX}^{\mathrm{e}}$ et $\mathrm{XX}^{\mathrm{e}}$ siècles $^{3}$, mais aussi à l'incroyable vivacité de ce champ de recherche dans de nombreux pays européens ${ }^{4}$. La France est, par ailleurs, peu présente dans les programmes européens de recherche en histoire de l'enseignement supérieur, la participation française à la Commission internationale d'histoire des universités, rattachée au Comité international des sciences historiques, n'étant le fait que d'historiens médiévistes ${ }^{5}$.

Cette faiblesse générale peut sans doute s'expliquer par une pluralité de facteurs. La rupture révolutionnaire, qui a brisé la continuité avec les institutions d'Ancien Régime, a eu pour effet de rendre difficile, méthodologiquement et intellectuellement, la production d'une histoire linéaire de ces institutions. Dans le même temps, le développement d'un système dual faisant cohabiter des grandes écoles dotées d'une forte identité (et s'intéressant souvent à leur propre histoire) et des universités dont la réalité institutionnelle, le rayonnement culturel et scientifique et la fonction sociale ont beaucoup varié, a contribué à complexifier le paysage. Par ailleurs, la rareté des services d'archives au sein des institutions d'enseignement supérieur a certainement renforcé cette difficulté, en ne permettant pas la production de séries archivistiques stables, organisées et accessibles ${ }^{6}$. Mais ce sont sans doute la forme très centralisée du système

3 Cf. Robert D. Anderson, European Universities from the Enlightenment to 1914, Oxford, Oxford University Press, 2004.

4 Il n'existe pas en France de revue consacrée à l'histoire de l'enseignement supérieur comme c'est le cas dans les pays anglo-saxons (History of universities, Perspectives on the history of higher education) ou en Italie (Annali di storia delle università italiane). Il n'existe pas non plus de centre de recherche spécialisé comme en Italie, en Belgique, aux États-Unis ou encore en Finlande ; pas plus que d'opérations éditoriales d'envergure, comme l'est, par exemple, la publication de The History of the University of Oxford (Oxford University Press) en 8 volumes entre 1984 et 1997.

5 L'enseignement supérieur à la période moderne souffre d'un même déficit. Si une série de travaux ont été publiés entre le milieu des années 1980 et la fin du siècle (citons, par exemple : Roger Chartier, Jacques Revel, Dominique Julia, Les Universités européennes du XVI ${ }^{e}$ au XVIII ${ }^{e}$ siè- $^{-}$ cle. Histoire sociale des populations étudiantes, Paris, Éditions de l'École des hautes études en sciences sociales, t. 1, 1986 ; t. 2, 1989 ; ou François Cadhilhon, Jean Mondot, Jacques Verger (dir.), Universités et institutions universitaires européennes au XVIII siècle. Entre modernisation et tradition, Bordeaux, Presses universitaires de Bordeaux, 1999), très peu de chercheurs s'intéressent actuellement aux universités d'Ancien Régime et la seule publication récente à son sujet est la thèse de Boris Noguès publiée sous le titre : Une Archéologie du corps enseignant. Les professeurs des collèges parisiens aux XVII et XVIII ${ }^{e}$ siècles (1598-1793), Paris, Belin, 2006, qui traite des professeurs de la faculté des arts de l'université de Paris. Une réflexion est cependant engagée par les médiévistes et les modernistes français dans la perspective de penser " une histoire renouvelée des université ", intégrant la longue durée. On peut en trouver le compte rendu sur le site du Centre de recherches d'histoire moderne (Université Paris I) : <http://crhm.univ-paris 1.fr/activites/hst_univ/resume.pdf>.

6 Cf. Emmanuelle Picard, "Les archives de l'enseignement supérieur français à l'époque contemporaine : un chantier d'avenir ? ", in Laurent Rollet (dir.), Mémoire et culture matérielle de l'Université, Nancy, Presses universitaires de Nancy, 2008, p. 51-62. 
mis en place dans le cadre de l'Université impériale, qui incluait le secondaire et le supérieur dans un même ensemble administratif, et la persistance de cette forme à travers une succession de régimes politiques très différents, qui sont principalement à l'origine de cette carence des recherches sur l'histoire des universités. Comme l'a montré Christine Musselin dans La Longue marche des universités françaises ${ }^{7}$, le dispositif d'enseignement supérieur français est organisé selon une logique verticale, autour des disciplines, et non horizontale, autour des établissements. Il n'existe donc pas de véritable culture d'établissement quand, au contraire, les disciplines académiques en viennent à jouer le rôle de régulateurs institutionnels. Il n'est donc pas étonnant que ces dernières soient davantage l'objet de recherches que les établissements universitaires eux-mêmes.

On dispose de quelques bonnes synthèses françaises sur l'enseignement supérieur, malheureusement un peu anciennes pour la plupart ${ }^{8}$. Plus récemment, la perspective comparatiste mise en œuvre par des historiens étrangers, ou sous leur direction, a permis la publication, de deux sommes dans lesquelles le cas français est largement traité : l'ouvrage de Robert Anderson sur les universités européennes au XIX ${ }^{\mathrm{e}}$ siècle ${ }^{9}$, et le troisième volume de A History of the University in Europe, sous la direction de Walter Rüegg ${ }^{10}$. L'intérêt majeur de ces travaux réside dans la mise en perspective des modèles napoléonien et humboldtien qui s'imposent alors aux universités européennes comme les voies modernes de la réforme de l'enseignement supérieur. Robert Anderson, en particulier, analyse précisément les effets de concurrence, mais aussi d'hybridation, qui sont alors à l'œuvre dans la plupart des pays européens, et remet en cause l'idée que la France ou l'Allemagne auraient offert une application exemplaire de l'un ou l'autre de ces modèles. Il propose également une réflexion sur les liens qu'entretiennent ces réformes et les projets politiques et

7 Paris, Presses Universitaires de France, 2001.

8 Antoine Prost, Histoire de l'enseignement en France 1800-1967, Paris, A. Colin, 1968 ; et aussi Éducation, société et politiques : une histoire de l'enseignement en France de 1945 à nos jours, Paris, Seuil, 1992 ; Louis-Henri Parias (dir.), Histoire générale de l'enseignement et de l'éducation en France, t. 3, De la Révolution à l'école républicaine, par Françoise Mayeur, et t. 4, L'École et la famille dans une société en mutation, par Antoine Prost, Paris, Nouvelle Librairie de France, 1981, rééd. Perrin, 2004 ; Jacques Verger (dir.), Histoire des universités en France, Toulouse, Privat, 1986 ; Christophe Charle, Jacques Verger, Histoire des universités, Paris, PUF, 1994 (rééd. 2007), coll. "Que sais-je ?".

9 R. D. Anderson, op. cit.

10 Universities in the Nineteenth and Early Twentieth Centuries (1800-1945), Cambridge, Cambridge University Press, 2004. Un dernier volume traitant de la deuxième moitié du $\mathrm{XX}^{\mathrm{e}}$ siècle reste à paraître. 
sociaux dans lesquels elles s'inscrivent. Enfin, l'élargissement de la perspective permet de poser la question de la transition entre l'Ancien Régime et la période contemporaine. Les réformes du début du XIX ${ }^{e}$ siècle prennent racine dans les transformations de la seconde moitié du siècle précédent, ce qui conduit à relativiser leur caractère de rupture et à faire apparaître les éléments de continuité.

Reste que, quelle que soit leur qualité, ces synthèses sont tributaires des travaux existants. Elles reflètent de facto les choix historiographiques de ceux qui ont travaillé sur l'histoire de l'enseignement supérieur, privilégiant certains moments (la Troisième République) et certaines approches (la sociologie du corps enseignant, en particulier). En effet, si certaines périodes sont aujourd'hui bien étudiées, d'autres constituent autant de terres à défricher. Les décennies qui séparent la fin du Premier Empire des débuts de la Troisième République restent mal connues, l'essentiel des informations dont nous disposons à leur sujet provenant de travaux anciens, et en particulier du livre de Louis Liard publié à la fin des années $1880^{11}$, enrichi, plus récemment, par des ouvrages sur l'organisation et l'administration de l'Instruction publique ${ }^{12}$. Ce traitement de défaveur renvoie à la doxa longtemps admise selon laquelle, avant la réforme de la Troisième République, les facultés françaises n'étaient pas à la hauteur du modèle humboltdien, qui mêlait étroitement enseignement et recherche. A contrario, les moments de transformation institutionnelle ont fait l'objet de nombreux travaux. Les années de fondation (1802-1815), en particulier, ont été étudiées pour interroger les ruptures et les continuités repérables dans l'œuvre napoléonienne ${ }^{13}$. La période républicaine (des années 1870 aux années 1890) est bien connue également, notamment grâce au livre (non traduit en français) de George Weisz, The Emergence of Modern Universities in France ${ }^{14}$ : c'est sans conteste

11 L'Enseignement supérieur en France, 1789-1889, Paris, A. Colin, 1888-1894.

12 Par exemple, Paul Gerbod, "L'administration de l'enseignement supérieur public en France de la Révolution à nos jours ", La Revue administrative, n² 230, 1986. Ou Guy Caplat et Bernadette Lebedeff-Choppin, L'Inspection générale de l'enseignement supérieur au XIXe siècle, Paris, INRP, 2002 ; et Jean-François Condette, Les Recteurs d'académie, t. 1 : La fabrication d'une élite administrative au service de l'Instruction publique, Lyon, INRP, 2006.

13 À ce sujet, voir, par exemple, Philippe Savoie, "L'Université impériale, le corps enseignant et l'institution scolaire publique. Origine et postérité d'une fondation ambiguë ", in Armelle Le Goff (dir.), Les Hommes et les femmes de l'Université. Deux siècles d'archives, Paris/Lyon, DAF/INRP, 2009, p. 11-23.

14 The Emergence of Modern Universities in France: 1863-1914, Princeton, N.J./Guildford, Princeton University Press, 1983. 
sur ces deux décennies que les publications sont les plus nombreuses. Enfin, si quelques monographies traitent de l'entre-deux-guerres, la période qui s'ouvre en 1945 pourrait être qualifiée de continent vierge. Seul l'épisode de 1968 a fait l'objet d'ouvrages, qui portent sur des aspects très limités, traitant essentiellement des mouvements étudiants. Ni la genèse de la loi Edgar Faure, ni sa mise en œuvre concrète n'ont été véritablement étudiées. Les bouleversements de la démographie enseignante et étudiante et leurs effets sont relativement mal connus. En revanche, les travaux portant sur l'enseignement supérieur depuis le début des années 1990 se sont développés en grand nombre en sociologie, en science politique et en géographie, en particulier autour des effets de la politique mise en œuvre dans le cadre des plans Universités 2000 et U3M, puis des transformations liées à la politique européenne de l'enseignement supérieur ${ }^{15}$.

Ce numéro d'Histoire de l'éducation voudrait présenter cette historiographie à la fois très segmentée et très hétérogène en mettant en lumière les incontestables apports des décennies passées, les chantiers en cours et les grandes orientations que pourrait prendre la recherche dans les années à venir. L'ambition de ce dossier est davantage de comprendre les lignes principales de l'historiographie que de proposer un recensement exhaustif des travaux. Le nombre des références bibliographiques a donc été volontairement limité. Une attention particulière a été portée à trois grands domaines actuellement en plein renouvellement : l'enseignement scientifique, l'histoire des femmes et la question de l'enseignement colonial.

Il convenait tout d'abord de porter un regard rétrospectif et analytique sur les travaux des quatre dernières décennies, afin d'éclairer les principaux choix méthodologiques et thématiques et de comprendre leurs incidences sur les recherches actuelles. Ainsi, la prééminence des questions relatives à l'histoire sociale est très nette dans les années 1970 et 1980, puis celles-ci cèdent la place à une approche centrée sur une lecture " culturelle " des questions d'enseignement supérieur (l'histoire des disciplines académiques) avant de voir se développer, plus récemment, un intérêt pour les effets de système, de mode d'organisation et de structuration du dispositif dans son ensemble. La présentation de cette construction, à la fois chronologique et thématique, des

15 On peut renvoyer en particulier aux travaux de Christine Musselin et de ses élèves ; ou pour les géographes, par exemple, à ceux de Myriam Baron. La bibliographie est riche et on peut en prendre connaissance par le biais de l'annuaire de chercheurs affiliés au RESUP (Réseau d'étude sur l'enseignement supérieur) : <http://www.resup.u-bordeaux2.fr/annuaire/gestionannuaire.htm>. 
champs de recherche fait l'objet du premier article du numéro, qui plaide pour une approche globale de l'histoire de l'enseignement supérieur français ${ }^{16}$.

La périodisation historiographique décrite dans ce premier article a par ailleurs largement à voir avec le développement des Science Studies anglosaxonnes, qui ont fait du cas français l'un de leurs terrains de prédilection. Fabien Locher montre dans son texte combien ces approches ont contribué à féconder un ensemble de recherches collectives et individuelles et à renouveler les paradigmes de la recherche ${ }^{17}$. C'est particulièrement le cas de la réflexion en termes d'approche locale, réflexion d'abord développée par des chercheurs anglo-saxons avant de connaitre une nouvelle impulsion avec les travaux d'historiens et de sociologues français ${ }^{18}$. L'ensemble de ces apports a contribué à développer un questionnement plus large sur les relations entre le centre et la périphérie, interrogeant la réalité des pratiques au regard de la norme réglementaire nationale, mais posant aussi la question des frontières entre niveaux (secondaire-supérieur) et types d'enseignement (général, classique, technique). Cette réflexion s'applique particulièrement au cas de "l'enseignement supérieur colonial ", présent en métropole comme catégorie spécifique et, de façon inégale, dans les pays colonisés ${ }^{19}$. Enfin, un dernier chantier en cours relève de la question du genre et porte sur la place des femmes dans l'enseignement universitaire français, considérée du côté des étudiantes, les enseignantes du supérieur étant encore aujourd'hui terra incognita ${ }^{20}$.

Emmanuelle PICARD Service d'histoire de l'éducation (INRP) emmanuelle.picard@inrp.fr

$16 C f$. Emmanuelle Picard, "L'histoire de l'enseignement supérieur français : pour une approche globale".

$17 C f$. Fabien Locher, "Les sciences et les techniques dans l'enseignement supérieur français (XIX'-XX' siècles) : un panorama historiographique".

$18 C f$. Laurent Rollet, "Peut-on faire l'histoire des pôles scientifiques ?".

19 Voir l'article de Pierre Singaravélou, "L'enseignement supérieur colonial" : un état des lieux ".

20 Voir l'article de Natalia Tikhonov Sigrist, " Les femmes et l'université en France, 1860-1914 : pour une historiographie comparée". 